Horizons philosophiques

\title{
Les dominicains et l'import-export : psychanalyse et existentialisme au Québec
}

\section{Yvan Cloutier}

Volume 2, numéro 1, automne 1991

De Buenos Aires à Québec

URI : https://id.erudit.org/iderudit/800887ar

DOI : https://doi.org/10.7202/800887ar

Aller au sommaire du numéro

Éditeur(s)

Collège Édouard-Montpetit

ISSN

1181-9227 (imprimé)

1920-2954 (numérique)

Découvrir la revue

\section{Citer cet article}

Cloutier, Y. (1991). Les dominicains et l'import-export : psychanalyse et existentialisme au Québec. Horizons philosophiques, 2(1), 91-105.

https://doi.org/10.7202/800887ar d'utilisation que vous pouvez consulter en ligne.

https://apropos.erudit.org/fr/usagers/politique-dutilisation/ 


\section{Les dominicains et l'import-export : psychanalyse et existentialisme au québec ${ }^{1}$}

II me paraît qu'on maltraite un peu en France les pensées et les bourses; on craint l'exportation du blé et l'importation des idées.

Voltaire $^{2}$

L'importation désigne, selon le Grand Larousse de la langue française ${ }^{3}$, l' "[a]ction d'introduire quelque chose d'étranger, de l'adopter". L'import/export, qu'il s'agisse de mode, de mots, de produits de consommation, de technologie, implique le déplacement d'un produit d'un territoire (national) à un autre, ce produit étranger étant susceptible de devenir un produit domestique. La matrice économique du concept sied-elle à la circulation des idées? L'homologie

1. L'article été écrit dans le cadre de Groupe de Recherche sur l'édition littéraire au Québec à l'université de Sherbrooke. Nous poursuivons ces travaux grâce à une subvention du Fonds FCAR à titre de chercheur collégial.

2. Voltaire, Lett. Chabaum, 28 septembre, 1770.

3. En six volumes, Paris, Librairie Larousse, 1973, t. 3. 
voltairienne entre blé et idées suppose l'existence d'un marché des biens symboliques avec sa logique spécifique. De plus, ce concept d'import/export suppose une territorialisation de la pensée, thèse qui ne va pas de soi en particulier lorsqu'il s'agit de discours à prétention universelle.

Les supposés d'un marché symbolique et de la territorialisation interrogent non seulement la légitimité des discours à prétention universelle, telle la philosophie, qui fondent justement leur légitimité sur leur «universabilité», mais aussi le statut des intellectuels. Car du coup l'intellectuel, qu'il soit producteur ou «illustrateur», opère dans le champ intellectuel de son territoire propre, lieu lui-même en rapport avec le champ intellectuel et le champ disciplinaire du territoire producteur ou récepteur. Selon que nous considérons la territorialité comme accidentelle ou comme structurelle (et structurante), nous apprécierons différemment les effets du double conditionnement territorial et par conséquent nous accorderons plus ou moins d'importance et de crédibilité à l'étude de ces conditionnements institutionnels.

II nous faut dès lors statuer sur la territorialité de la pensée. Je me limiterai ici à l'étude de la circulation de deux produits, soit la psychanalyse et l'existentialisme, dans le champ canadien-français des années 40-60 et plus spécifiquement au rôle des dominicains pour Montréal et Ottawa ${ }^{4}$. Notre étude vise aussi à valider la notion d'،instrumentalisation" dans la question des produits symboliques. L'existentialisme et la psychanalyse permettent d'établir que la circulation d'un produit symbolique se fait selon un processus d'،instrumentalisation", c'est-à-dire que l'usage d'un tel bien est déterminé par la dynamique du champ récepteur. Dès lors, la territorialisation agit tant au niveau de la prise en charge que dans ce que l'on en

4. Une autre communauté religieuse, les oblats de Marie-Immaculée, joue un rôle important dans la réception de la psychanalyse et de l'existentialisme au Québec via l'Université d'Ottawa. 
fait; en d'autres termes, l'usage obéit à des ancrages dans le champ et opère une sélection/adaptation des contenus. En conclusion, j'indiquerai quelques conséquences de mes avancées : abandon du cliché de l'assimilation métropolepériphérie et contenu original-dégradé, abandon de la thèse de l'aliénation qui lui a été associée et nécessité d'élargir les critères d'évaluation de la fécondité de l'usage d'un bien symbolique.

\section{L'hybridité territoriale des communautés religieuses.}

Les dominicains, au même titre que les autres communautés dont la maison-mère est à l'étranger, sont conditionnés par plusieurs territoires : le Québec de par le lien au couvent, à Ottawa de par leur appartenance à la province, à la France de par les rapports avec la maison-mère et lieux de formation et de publication, et enfin à Rome de par le maître général. Cette hybridité constituera un atout ou un handicap selon le caractère progressif ou conservateur de ces lieux.

Les communautés ont le privilège de sanctionner elles-mêmes les compétences. En effet, elles assurent la formation de leur membre du noviciat (même souvent dès le primaire) aux études universitaires, sans oublier dans le cas des dominicains leur propre hiérarchisation (lecteur en théologie et le prestigieux titre de sacré maître en théologie). Leur accès à d'autres territoires leur permet de mobiliser rapidement des compétences dans des secteurs en forte croissance; ce que les dominicains firent au Canada en important des experts sans lesquels l'Institut d'études médiévales et la Faculté des sciences sociales n'auraient pu acquérir si rapidement leur renommée.

Elles disposent de ces autres mécanismes de sanction et de promotion des compétences que sont les lieux d'édition. Ainsi les dominicains, qui s'auto-éditaient déjà sous divers lieux d'édition, croient bon, dès 1931, de créer l'CEuvre de Presse dominicaine pour coordonner les activités éditoriales (Revue Dominicaine, Le Rosaire, les bul- 
letins paroissiaux et les monographies). En 1937, ils fondent les Éditions du Lévrier. En outre, les dominicains jouissent d'une filière française en Europe qui leur permet de publier dans des revues françaises ou belges.

Les Éditions du Lévrier éditent ou coéditent leurs propres auteurs et des auteurs européens grâce à des ententes avec les Éditions du Cerf (maison dominicaine française), les Éditions Spes, les Éditions Lethielleux, Téqui, la Librairie du Recueil Serey, la Librairie philosophique Vrin, Desclée de Brouwer, les Éditions de la Lyre et de la Croix. Pendant la guerre, ils se sont aussi prévalus d'un arrêt exceptionnel visant les biens ennemis pour ajouter à leur catalogue des titres de Flammarion, des Éditions de la Vie spirituelle, de Desclée de Brouwer et de la Librairie Lecoffre; les éditeurs devaient verser au Séquestre un tantième de un pour cent du prix de détail des titres imprimés. Éditions et coéditions permettent aux dominicains de se constituer rapidement un catalogue prestigieux, et par là de promouvoir l'«image de marque" de la communauté tout en lui ouvrant un marché extérieur sans lequel l'édition ne pouvait être rentable.

L'autre avantage dont jouissent les intellectuels religieux est le fait que l'approbation de l'ordinaire (imprimi potest) soit précédée par la sanction du provincial (imprimi potest) sur recommandation de deux autres censeurs appartenant à la même communauté (nihil obstat) et d'un autre évaluateur de la communauté, ce qui n'est pas sans exercer une pression sur l'ordinaire, considérant les compétences des premiers évaluateurs. Les revues étaient publiées auctoritatum permissu.

Le rapport d'autorité de l'évêque sur le religieux est médiatisé par la communauté locale, elle-même sous l'autorité de territoires exogènes (le Prieur et le Maître général) sans oublier les rapports politiques qui pouvaient s'instaurer à divers niveaux. Dans le cas des dominicains, le Doyen et le secrétaire de la Faculté de philosophie de l'Université de Montréal, ainsi que les directeurs des insti- 
tuts rattachés étaient des dominicains. Dès lors, ils pouvaient exercer un contrôle sur les programmes et le choix des enseignants pour la plupart dominicains ou provenant de communautés alliées ou de laïcs "sûrs". En cas de conflit, l'autorité ecclésiale devait suivre la filière Père provincial, Doyen, professeur, et dans certains cas la filière française voire même la filière romaine. Par exemple le Père G.-H. Lévesque se serait-il sorti indemne des procès qu'on lui a fait à Rome s'il avait été membre du clergé régulier? De même, le Père Marie-Ceslas Forest et le Père Mailloux pouvaient compter sur des appuis à Rome dans leur démarche pour faire accepter le programme de l'Institut de psychologie. D'ailleurs, les dominicains rattachent leurs instituts et écoles à des Facultés de philosophie (l'institut d'Études médiévales et l'Institut de psychologie à Montréal et l'École des sciences sociales à Québec) afin de les protéger, grâce au statut canonique des institutions philosophiques, des incursions des autorités universitaires et des autorités politiques ${ }^{5}$.

En outre, nous connaissons les rapports privilégiés qu'entretenaient les dominicains avec des dirigeants politiques canadiens et avec le personnel diplomatique français à Ottawa. Le Père Pie-Marie Gaudrault, o.p. comptait "parmi ses intimes, je cite le Père G.-H.Lévesque ${ }^{6}$, des personnalités politiques de premier plan, de même que des ecclésiastiques distingués, comme par exemple les délégués apostoliques Andrea Cassulo et lldebrando Antoniutti", mais aussi Henri Bourassa, Ernest Lapointe et le sénateur Dandurand. Guy Sylvestre, critique très influent

5. Pour l'Institut de psychologie, voir les mémoires du Père Forest dans Yvan Lamonde et Benoît Lacroix, "Les mémoires du doyen Ceslas Forest, o.p.", Philosophiques, vol. III, $n^{\circ} 1$, p. 68.

Pour l'École des sciences sociales, voir Marcel Fournier, «Les conflits de discipline : philosophie et sciences sociales au Québec, 1920-1960", dans Philosophie au Québec, Montréal, Bellarmin, 1976, p. 218.

6. Georges-Henri Lévesque, Souvenances 1-entretiens avec Simon Jutras, Montréal, Éditions de la Presse, 1983, p. 141. 
et secrétaire de Louis Saint-Laurent, résidait au couvent de Montréal lors de ses visites à Montréal.

Cette hybride appartenance à plusieurs territoires représentait une menace pour les évêques. Ce n'est pas pour rien que les dominicains durent s'implanter d'abord à Sainte-Hyacinthe, et qu'il fallut beaucoup de temps et de diplomatie pour qu'ils puissent s'établir à Montréal, à Québec et à Ottawa. Sont aussi connus les rudes conflits entre l'Évêque de Montréal et les sulpiciens. Le pouvoir de la communauté peut aussi tirer profit des alliances entre communautés, je pense ici aux rapports entre les sulpiciens et les dominicains. II ne faut pas oublier que la plupart des communautés sont venues de la France dans des visées expansionnistes qui les mettaient en concurrence pour le recrutement (le Québec étant une terre fertile) et pour l'exercise des divers apostolats. Ainsi les oblats s'objectèrent à la venue des dominicains à Ottawa car l'enseignement universitaire à Ottawa leur appartenait.

L'hybridité territoriale a permis à une communauté peu nombreuse, 80 en 1931, et en plus suspecte à cause de sa réputation libérale, d'imposer sa présence d'abord dans le champ philosophique et de là dans le champ nouveau des sciences humaines et des sciences sociales.

\section{Hybridité territoriale et champ intellectuel}

Comment cette structure territoriale agit-elle dans le fonctionnement du champ intellectuel? Elle opère sur deux processus : le processus d'IMPORTATION et le processus de LÉGITIMATION.

Nous proposons trois étapes dans le PROCESSUS D'IMPORTATION : (1) la SAISIE ou l'entrée dans le territoire propre par les activités de $(1 \mathrm{~A})$ formation (à l'étranger ou ici par le recours aux intellectuels-experts et à l'importation du livre), (1B) l'importation d'intellectuels-experts qui deviennent des diffuseurs; (2) la DIFFUSION par (2A) l'enseignement, (2B) la formation de groupes de discussion, de colloques, (2C) par l'activité éditoriale. 
Dans ce processus de circulation intervient un processus de LÉGIMITATION qui s'inscrit dans les structures territoriales; ce processus utilise les cautions provenant d'un autre territoire. Il y a d'abord (a) l'approbation par une autorité exogène pouvant s'exercer ici, que ce soit (aa) une autorité qui a un droit d'intervention reconnu (ex. droit canonique, droit international), ou (ab) une compétence (institution ou individu) dotée du pouvoir de consécration (académies, prix littéraires de la métropole culturelle, directeur de collection dans une maison d'édition reconnue). La diffusion d'une oeuvre autochtone à l'étranger peut être aussi utilisée comme un indicateur de légitimité.

Nous nous proposons maintenant d'illustrer ce processus par la réception de la psychanalyse et de l'existentialisme dans le Québec des années 40-60. Nous devons nous limiter ici à présenter des jalons d'une telle description.

\subsection{La réception ${ }^{7}$ de la psychanalyse}

Le processus d'import de la psychanalyse par les dominicains suit les trois étapes ci-haut décrites. Dès 1931, le Père Ceslas-M. Forest o.p., doyen de la Faculté de philosophie de l'Université de Montréal, a le projet de mettre sur pied un institut de psychologie qui mettrait de l'avant l'enseignement et la recherche en psychologie expérimentale. A cet effet, ne pouvant trouver un dominicain disponible, il demande à l'abbé Irénée Lussier (futur recteur) d'étudier en psychologie et il lui offre un cours de psychologie expérimentale à la Faculté de philosophie en $1934^{8}$. Le Père Marcel-Marie Desmarais, qui avait étudié la psychologie à Paris, publie un long texte sur la psychanalyse dès 1936 dans le premier Cahier Philosophie de la collec-

7. Le mot “réception" a l'avantage de désigner tant l'entrée dans le territoire que la modalité de cet accueil (sélection des contenus, jugements de valeurs).

8. Yvan Lamonde et Benoît Lacroix, «Les mémoires...», p. 70. 
tion "Études et recherches". II faut cependant rappeller que la Revue dominicaine avait publié en 1930 et 1931 des chapitres de la thèse de doctorat du Dr Antonio Barbeau soutenue à la Faculté de philosophie en 1930 et intitulée "Les bases du freudisme». Ce Barbeau, formé en France et aux États-Unis, enseignera à l'Institut de psychologie, où il organise l'enseignement de la psychophysiologie; il préside la Société de philosophie de Montréal en 1936.

II faudra attendre 1942 pour que l'Institut voit le jour. Les annuaires 1944-1945 et 1946-1947 indiquent un cours de $3^{e}$ année intitulé «S. Freud" (20 leçons) et un cours de $4^{\mathrm{e}}$ année, «Le système psychologique de Freud» enseigné par le dominicain Augustin Deslauriers. Le procès-verbal de la réunion du 16 mars 1945 contient une recommandation en vue de la nomination au titre de professeur agrégé du Dr Barbeau et du Dr Prados (titulaire de l'Université de Barcelone et professeur de psychiatrie à l'Université McGill). Nous savons, d'après les procès-verbaux de la Faculté, que Théo Chantrier a enseigné à l'Institut à tout le moins en 1950-1951. André Lussier y enseignera en 1948 et sera nommé agrégé en février 1951.

Mais le grand médiateur de la psychanalyse est le Père Mailloux comme le rappelle André Lussier ${ }^{10}$ et $M$. Prados dans son histoire de la psychanalyse à Montréal publiée dans La Revue Canadienne de Psychanalyse ${ }^{11}$. II engage André Lussier. pour dispenser un cours sur la psychanalyse à l'Institut; il est aussi responsable de la venue à Montréal du psychanalyste Théo Chantrier et Zilboorg. Mailloux qui faisait lui-même des thérapies psychanalyti-

9. D'après : [collectif], "À la mémoire du professeur Antonio Barbeau», Le Journal de l'Hôtel Dieu, p. 211-293. Je tiens cette information de Michel Clément.

10. André Lussier, "Le "feu sacré" : de la psychologie à la psychanalyse", dans La naissance de la psychanalyse... à Montréal, Frayages, $n^{\circ} 3$, Montréal, Société d'éditions Frayages, 1987, p. 27-46.

11. Volume 1, 1954, p. 3-33. 
ques, tout comme Deslauriers, crée un Cercle de psychanalyse dont sortira la Société canadienne de psychanalyse.

Les dominicains doivent utiliser tous leurs appuis, Mgr Charbonneau à Montréal, le Maître général à Rome, pour réussir à obtenir de Rome l'approbation du programme de l'Institut de psychologie qui était bloquée à cause des recherches et des enseignements sur la méthode expérimentale et sur la psychanalyse que les dominicains classaient dans les psychologies expérimentales.

Mailloux, Deslauriers et les autres Dominicains qui allaient se joindre à eux autour du Centre de recherches en relations humaines (Dominique-H. Salman, Bernard Mailhot et, plus tard, Claude-François Lévesque) tiraient une caution du côté de catholiques qui oeuvraient dans la recherche en psychologie expérimentale : le Cardinal Mercier, l'École de Milan et le Père Plé et de sa revue Vie Spirituelle. Mailloux avait lui-même étudié cette discipline à Rome. Les Éditions du Cerf, propriétés des dominicains français, publient trois livres qui ont des rapports avec la psychanalyse et le Québec : (1) la traduction de G. Zilboorg, Sigmund Freud et l'activité mentale de l'homme (1957); ce converti était dans l'entourage de Mailloux et associé à ses travaux. (2) J.R. Dempsey, Freud, psychanalyse et catholicisme (1955); l'auteur a soutenu à l'Université de Montréal une thèse de doctorat The Psychology of Jean-Paul Sartre (1948-1949); il écrit à la page 8 : "Au Canada français, à l'Institut de psychologie de l'Université de Montréal, il semblait possible d'intégrer les plus importantes découvertes de Freud au sein du thomisme»; et (3) Henri Gratton, Psychanalyses d'hier et d'aujourd'hui (1955) des recherches en psychologies expérimentales; cet oblat enseignait à Ottawa.

\subsection{La réception de l'existentialisme}

Encore ici, les dominicains jouent un rôle de médiateur. Quoique les journalistes et critiques aient été les médiateurs de Sartre par le succès médiatique qu'ils avaient 
contribué à créer à l'occasion de Huis clos en fin de janvier 1946, les dominicains, par l'intermédiaire du Père Forest, ne tardèrent pas à entrer en scène. En effet, réalisant que Sartre était un philosophe athée, une réaction hostile s'organise autour du Devoir qui repique des articles de la revue des jésuites français Études et de d'autres revues. Le comité organisateur de la Société d'étude et de conférences, qui avait invité Sartre à son thé-causerie, envisage d'annuler la conférence de Sartre. Mais, sur le conseil de son directeur spirituel, le Père Forest, le comité demande conseil à Mgr Charbonneau qui donne son accord. Cette conférence, organisée par Radio-Canada et largement couverte par les journaux, contribua largement à la diffusion de l'existentialisme sartrien.

Jacques Lavigne enseigne Sartre à la session de l'hiver 1947 dans le cadre d'un cours d'histoire de la philosophie. Prétextant la popularité de l'existentialisme, le doyen Forest demande à l'abbé Jean Milet de donner un cours sur Sartre; ce qui fut fait l'année 1951-195212. Parmi les étudiants : Hubert Aquin. Michel Roy ${ }^{13}$ rappelle que les professeurs parlaient de Sartre et Camus; lui-même fit un mémoire de maîtrise sous la direction du Père Louis Lachance. Nous rappelons la thèse de Pierre Dempsey, o.f.m. The Psychology of J.-P. Sartre (1948-1949). Sartre est le quatrième parmi les auteurs dont les noms apparaissent le plus souvent dans les titres de mémoires et de thèses de 1940 à 1971; avec ses 14 mentions (ex aequo avec Bergson), il suit Thomas d'Aquin (42), Gabriel Marcel (20) et Maurice Merleau-Ponty (15).

Les dominicains français s'étaient engagés tôt dans un dialogue avec l'existentialisme athée. Je pense à la discussion sur le péché (publiée dans Dieu vivant de 1945)

12. Le même Milet consacre à Sartre une section d'un cours d'Histoire de la philosophie en 1950-1951.

13. Yvan Cloutier, "Le Québec des années Quarante et Cinquante. Entretien avec Michel Roy», Philosopher, n 8, 1989, p. 35-43. 
à laquelle participent Sartre, Marcel, Bataille et les dominicains D. Dubarle et Maydieu, et au numéro spécial de la Revue de philosophie (1946) intitulé "L'existentialisme» avec des articles des dominicains F. Cayré, P. Descoqs et D. Dubarle. Benoît Pruche, o.p. publie Existentialisme et acte d'être en 1947 et L'homme de Sartre en 1949 chez Arthaud. Le Père H. Paissac publie Le dieu de Sartre (1950) dans la même collection. Le même Pruche, sur une proposition du doyen Forest, est admis "en $4^{e}$ année de la Faculté de philosophie en vue du doctorat. Le Conseil approuve le projet d'une thèse du $P$. Pruche : «Essai de philosophie existentielle d'inspiration thomiste : existant et acte d'être" (procès-verbal - Conseil de la Faculté du 15 septembre 1952). La thèse sera soutenue en 1957 sous le nouveau titre «Existant et acte d'être. Essai de philosophie existentielle»; le thomisme n'y est plus! Pruche publie deux articles sur l'existentialisme dans la Revue de l'Université d'Ottawa et un article intitulé "Sur la conscience de soi» dans le Cahier VIII de la collection Études et recherches (1952).

\section{L'instrumentalisation comme moteur du processus d'import}

Nous avons montré brièvement que les dominicains ont joué un rôle que nous croyons déterminant dans la réception de la psychanalyse; nous aurions pu ajouter les sciences sociales. Nous voulons insister sur un point : ce processus d'import implique certes un territoire étranger, voire même une métropole, mais l'activité d'import s'inscrit selon nous dans la dynamique interne du champ québécois. Si les dominicains importent la psychanalyse et l'existentialisme, c'est qu'ils y voient un instrument d'intervention dans un champ intellectuel qu'ils entendent modifier. II faudrait plutôt dire que les dominicains se servent de la psychanalyse et de l'existentialisme dans une visée apostolique. 
La crise économique avait entraîné une crise culturelle importante : critique du libéralisme, perte de confiance dans les institutions traditionnelles accompagnées d'une baisse de la pratique religieuse. L'essor de la radiophonie dans les années trente et de l'édition pendant la guerre avaient contribué à faire des années quarante une période d'ouverture, du moins avant 1949.

La crise entraîne deux phénomènes qui semblent à première vue contradictoires : d'abord, une centration de l'individu sur lui-même qui fait suite à l'effondrement des croyances - cette crise sera alimentée par la littérature psychologique de l'époque - et le besoin de trouver dans les sciences humaines des outils pour élaborer un autre lieu, une autre culture. Ce que certains, les gens de la Relève et les jésuites de l'École sociale populaire comprennent comme une crise spirituelle à laquelle il faut opposer une «médecine» spirituelle; d'autres, j'inclus les dominicains, développent un souci de scientificité et d'efficacité. Cette nouvelle mentalité favorise le développement rapide des sciences économiques, de la sociologie (urbanisation, mutations dans la paroisse), et, avec le développement du système scolaire, des sciences de l'éducation (orientation scolaire ${ }^{14}$, psychopédagogie).

Le clergé était aussi aux prises avec des problèmes de contamination de l'idéal ascétique; il fallait mieux s'équiper pour sélectionner, former et, dans certains cas, traiter les pathologies du sentiment religieux. Scientificité et efficacité allaient de pair avec le développement des sciences expérimentales.

Avant même que Sartre ne vienne au Québec ou que des psychanalystes étrangers viennent s'y établir, il y avait déjà une demande, créée par la crise, et une certaine présence ici de ces deux courants. Les dominicains ne peuvent développer ces courants ici que parce qu'il y a des 
ancrages. Ils se servent de ces courants, d'une part, comme réponse à une demande et, d'autre part, comme d'un levier pour accroître leur pouvoir symbolique.

Ce qui les intéresse, c'est certes les usages pratiques, i.e. thérapeutiques, mais aussi la méthode expérimentale qu'ils ne considerent pas comme plusieurs philosophes catholiques d'alors comme une menace; au contraire, leur foi dans la philosophie thomiste cautionne leur avancée dans la méthode expérimentale ${ }^{15}$. Ils organisent au début des années quarante des colloques et ils en publient les actes; ils savent attirer les compétences et ainsi s'assurer par là une hégémonie dans ces domaines qui allaient connaître un essor important.

Parlant de Ceslas-M. Forest, Jean Hamelin et Nicole Gagnon écrivent que «toute sa vie [il] aura "mené une lutte contre le crétinisme" des Canadiens français ${ }^{16}$ ". Les dominicains correspondent bien à la description que le sociologue Rocher donne de l'idéologie de rattrapage; il ne s'agit pas tant de rattraper la France que de mettre en place la superstructure qui corresponde aux mutations structurelles. A la stratégie de l'ignorance qui n'est plus possible, et à l'attaque frontale, ils opposent la connaissance compétente qui seule permet d'adapter ces connaissances et ces pratiques à la philosophie thomiste. Certains parleront de récupération, je préfère parler d'instrumentalisation : on se sert par exemple de la psychanalyse pour guérir après avoir libéré la psychanalyse de ses postulats matérialistes. Le Père Mailloux croyait même rendre service à la psychanalyse en la thomisant et rendre service au thomisme en l'enrichissant de confirmations et de prolongements expérimentaux.

15. Sur la thomisation des sciences psychologiques, voir Noël Mailloux, "Le deuxième anniversaire de l'Institut de psychologie de l'Université de Montréal", Contribution à l'Étude des sciences de l'homme, $n^{\circ} 2,1953$, p. 7-12.

16. Jean Hamelin et Nicole Gagnon, Le XX $X^{e}$ siècle, $t .1: 1898-1940$, Montréal, Boréal, 1984, p. 415. 
Quant à l'existentialisme, il avait ses connaisseurs et quelques adeptes bien avant le raz-de-marée existentialiste français de l'automne 1945 et montréalais du printemps 1946. Le Père R. Trudel o.m.i. donnait à Ottawa un cours sur les philosophies existentielles en 1940-1941; les étudiants, parmi lesquels Guy Sylvestre, Jacques Croteau et Gaston Carrière, avaient alors fait un inventaire de tous les livres sur la philosophie existentielle que l'on pouvait trouver dans les bibliothèques de la ville d'Ottawa. Gabriel Marcel avait déjà ses lecteurs, ses adeptes ${ }^{17}$, voire même ses correspondants. L'Annuaire (1945-46) de la Faculté de philosophie indique un cours, «Position et appréciation de l'existentialisme français", offert par Arcade Monette o.p., le même qui qualifie la philosophie de Maine de Biran de philosophie existentialiste dans "La théorie des premiers principes selon Maine de Biran ${ }^{18}$ ", soit avant la vague existentialiste parisienne de l'automne 1945 et québécoise du printemps 1946. Encore ici, il s'agit d'enrichir le thomisme qui demeure la philosophie qui peut fonder les sciences humaines et qui est lui-même une philosophie de l'existence.

\section{Conclusion}

Le concept d'hybridité territoriale permet, selon nous, de compendre la circulation d'une vision du monde d'une culture productrice à une culture réceptrice. Les communautés religieuses, à cause de leur hybridité territoriale, constituent un objet privilégié pour l'étude de la circulation des idées entre une culture périphérique et une métropole.

17. Sur les traces de Marcel au Québec dès la fin des années trente, voir Roland Houde, «Pour saluer Alexis Klimov - Reconnaissance de Marcel Raymond (1915-1972)", dans De la philosophie comme passion - Hommage à Alexis Klimov, Québec, Éditions du Beffroi, 1984, p. [171]-195.

18. Arcade Monette o.p., La Théorie des premiers principes selon Maine de Biran, éd. du Lévrier et Librairie philosophique J. Vrin, Montréal, Ottawa et Paris, 1945. 
La réception d'un bien symbolique renvoie certes à la culture productrice mais le processus a des conditions qui tiennent à la culture réceptrice. Le processus de circulation s'inscrit dans des structures territoriales et autochtones.

Nous nous sommes limité à une topique, c'est-à-dire à la circulation d'informations dans des lieux structurants. L'analyse institutionnelle doit cependant être complétée par l'analyse des contenus. En effet, nous croyons que la territorialisation opère par une sélection/adaptation des contenus. Enfin, il nous faut évaluer les effets de la réception non seulement en terme de reproduction mais d'effets sur les lieux structurants et de là sur les agents qui s'y inscrivent. La question n'est pas d'inventorier les existentialismes québécois ou les psychanalyses québécoises mais d'évaluer l'effet de ces courants sur les lieux dans lesquels se sont formés les intellectuels importants des années cinquante et soixante.

YVAN CLOUTIER Collège de Sherbrooke 